\title{
ASSESSMENT OF FINISH MACHINING AND MASS FINISHING AS POST- PROCESSING METHODS FOR PBF-LB/M-MANUFACTURED 316L
}

\author{
C. Fuchs ${ }^{1 *}$, L. Kick ${ }^{1}$, O. Leprevost ${ }^{1}$, M. F. Zaeh ${ }^{1}$ \\ ${ }^{1}$ Technical University of Munich, Department of Mechanical Engineering, Institute for Machine Tools and Industrial \\ Management, Boltzmannstrasse 15, 85748 Garching, Germany \\ ${ }^{*}$ Corresponding author; e-mail: christina.fuchs@iwb.tum.de
}

\begin{abstract}
Additive manufacturing techniques are increasingly used in industry. However, the direct usage of additively manufactured parts is limited due to their relatively low surface quality. Especially to achieve functional surfaces, post-processing has to be carried out. Post-processing methods include traditional mechanical cutting processes as well as electrical and chemical processes. Since previously deposited material is removed and additional manufacturing time is necessary, post-processing leads to increased manufacturing costs. Therefore, if additive manufacturing is to be competitive with traditional manufacturing processes, choosing the correct post-processing method is vital. Decision parameters, for example, are the achievable surface quality, the amount of material removal, and the preservation of the shape. In this article, the suitability of two traditional manufacturing processes, milling and mass finishing, as post-processing methods for parts from 316L, manufactured with powder bed fusion using a laser beam, is described. It is characterized how the depth of material removal influences the surface quality. For the milling process, it is determined that a depth of material removal of $0.2 \mathrm{~mm}$ leads to a stable surface quality. Finally, the processes' effectiveness as post-processing methods is assessed by comparing the achievable surface quality, showing that mass finishing processes are an economic postprocessing option in specific cases.

Keywords:
\end{abstract}

Post-Processing; Finishing; Vibratory Finishing; Additive Manufacturing; Machining Allowance

\section{INTRODUCTION}

Additive manufacturing techniques are used more and more in industry, and powder bed fusion of metals using a laser beam (PBF-LB/M) is one of these technologies. In the process, a layer of powder is applied by a coater. The laser beam melts the powder in the areas that will form the component. Then, the coater applies a new layer of powder. [Gibson 2015] However, the surface quality of the manufactured parts is often not sufficient [Kumbhar 2018]. For sealing surfaces with a static load, a surface roughness $R_{a}<3.2 \mu \mathrm{m}$ is required [Labisch 2014]. Such functional surfaces cannot be achieved with PBF-LB/M at the moment, with the surface roughness of as-built components ranging between 2 and $10 \mu \mathrm{m}$ [EOS GmbH; Kaynak 2019]. The parts' fatigue behavior is influenced by the surface roughness as well [Elangeswaran 2019]. Therefore, postprocessing is often carried out [Kumbhar 2018]. In recent years, various post-processing options have been researched for several materials. One of these materials is the austenitic steel alloy 316L. In Tab. 1, an overview of the state of the art on 316L's post-processing methods is given. The application of these processes is limited since the necessary amount of material removal to achieve these surface qualities has not been determined yet. The amount has to be determined to design components for PBF-LB/M and post-processing, so that it can be added as a machining allowance to the design. Therefore, within this study, it is characterized how the depth of material removal influences the surface quality for finish machining and mass finishing. Consequently, an analysis of the effectiveness of the processes as post-processing methods is given, taking the resulting surface quality and amount of material removal into consideration.

\section{METHODOLOGY}

To determine the influence of the depth of material removal on the surface roughness, finish machining and mass finishing experiments were carried out. In the following, the depth of material removal necessary to achieve a stable surface quality, with surface roughness parameters within the range for functional surfaces, is referred to as the necessary depth of material removal.

It was assumed that the necessary depth of material removal is dependent on the surface roughness of the as-built components. Finish machining can be performed via turning and milling. Therefore, its general process principle is that of cutting with a geometrically defined 
cutting edge. A tool with a defined number of cutting edges is used to separate material from a workpiece during finish machining, resulting in chips [Klocke 2014]. The amount of material removed thus depends on the path the tool takes. Theoretically, different depths of material removal are possible on different surfaces of one component. Therefore, PBF-LB/M process parameters influencing the surface quality were included in the research, and milling was chosen as the finish machining option.

Tab. 1: State of the art on post-processing methods for PBF-LB/M-manufactured $316 \mathrm{~L}$.

\begin{tabular}{|c|c|c|}
\hline process & $\begin{array}{c}\text { reduction } \\
\text { of surface } \\
\text { roughness } \\
R_{a} \text { in } \%\end{array}$ & $\begin{array}{c}\text { final } R_{a} \\
\text { in } \mu \mathrm{m}\end{array}$ \\
\hline $\begin{array}{l}\text { finish machining } \\
\text { [Kaynak 2018] }\end{array}$ & 88 & $<1.0$ \\
\hline $\begin{array}{l}\text { magnetic abrasive finishing } \\
\text { [Zhang 2019] }\end{array}$ & 75.7 & - \\
\hline $\begin{array}{l}\text { surface attrition treatment } \\
\text { [Sun 2019] }\end{array}$ & 96 & 1.8 \\
\hline $\begin{array}{l}\text { disk grinding } \\
\text { [Löber 2013] }\end{array}$ & - & 0.3 \\
\hline $\begin{array}{l}\text { blasting } \\
\text { [Löber 2013] }\end{array}$ & - & 3.9 \\
\hline $\begin{array}{l}\text { vibratory surface finishing } \\
\text { [Kaynak 2019] }\end{array}$ & 41.4 & 4.1 \\
\hline $\begin{array}{l}\text { drag finishing } \\
\text { [Kaynak 2019] }\end{array}$ & 52.9 & 3.3 \\
\hline $\begin{array}{l}\text { machining and polishing } \\
\text { [Afkhami 2021] }\end{array}$ & - & 1.5 \\
\hline
\end{tabular}

In contrast, during mass finishing, the material is removed by impact and relative movement between individual parts and an abrasive medium, resulting in shearing and a microscopic amount of material removal [Domblesky 2003]. Thus, the process is even applicable to microstructured parts as designed by [Calleja-Ochoa 2021]. Due to the process principle, different depths of material removal within one component are difficult or impossible to realize. Therefore, two mass finishing processes and their process parameters were studied without considering the PBF-LB/M parameters.

\subsection{Milling}

\section{Experimental procedure}

Based on the state of the art, influential parameters for the surface quality of PBF-LB/M-manufactured parts were determined. The orientation on the build platform [Hitzler 2017], the inclination angle of the surface [Kleszczynsk 2015], and the orientation of the surface, up- or down-skin, were chosen as factors for the research since they are considered to have a significant influence on the surface quality. The resulting twelve types of factor combinations and their designations are listed in Tab. 2.

The orientation on the build platform describes the placement of the samples' longitudinal axis in regard to the build platform and, thus, to the movement of the coater and gas flow. Two orientations, a rotation of the length axis by $45^{\circ}$ and a rotation by $135^{\circ}$, both clockwise from the horizontal, were chosen.

The second parameter was the inclination angle of the sample surfaces. The inclination angle is defined as the angle between the build platform and the surface. On simple parts, $0^{\circ}$ and $90^{\circ}$ angles are common. Therefore, both were considered in this research. Support structures were avoided due to their influence on the surface quality. Therefore, the minimum setting of $45^{\circ}$ was chosen [Thomas 2009]. A $67.5^{\circ}$ angle was chosen as the equidistant step between $45^{\circ}$ and $90^{\circ}$. For the $45^{\circ}$ and $67.5^{\circ}$ angles, two surface orientations exist: up-skin and down-skin. Since these surface orientations might influence the initial surface roughness, both were considered.

Tab. 2: Factor combinations for the milling experiments.

\begin{tabular}{llll}
\hline $\begin{array}{l}\text { combina- } \\
\text { tion } \\
\text { number }\end{array}$ & $\begin{array}{l}\text { orientation } \\
\text { on build } \\
\text { platform }\end{array}$ & $\begin{array}{l}\text { inclina- } \\
\text { tion } \\
\text { angle }\end{array}$ & $\begin{array}{l}\text { surface } \\
\text { orien- } \\
\text { tation }\end{array}$ \\
\hline M1 & $45^{\circ}$ & $0^{\circ}$ & - \\
M2 & $45^{\circ}$ & $90^{\circ}$ & - \\
M3 & $45^{\circ}$ & $45^{\circ}$ & down-skin \\
M4 & $45^{\circ}$ & $45^{\circ}$ & up-skin \\
M5 & $45^{\circ}$ & $67.5^{\circ}$ & down-skin \\
M6 & $45^{\circ}$ & $67.5^{\circ}$ & up-skin \\
M7 & $135^{\circ}$ & $0^{\circ}$ & - \\
M8 & $135^{\circ}$ & $90^{\circ}$ & - \\
M9 & $135^{\circ}$ & $45^{\circ}$ & down-skin \\
M10 & $135^{\circ}$ & $45^{\circ}$ & up-skin \\
M11 & $135^{\circ}$ & $67.5^{\circ}$ & down-skin \\
M12 & $135^{\circ}$ & $67.5^{\circ}$ & up-skin \\
\hline
\end{tabular}

For each of the twelve combinations, five samples were additively manufactured by FIT AG (Lupburg, Germany), and four samples were subsequently machined. One sample of each combination was kept in as-built condition to illustrate the difference between the surface roughness of as-built and machined components. The samples' geometry is depicted in Fig. 1. The width of the samples was $10 \mathrm{~mm}$, while the length and height varied between $10 \mathrm{~mm}$ and $39 \mathrm{~mm}$. All samples were produced in one build job. This way, the same process parameters were guaranteed for every component.

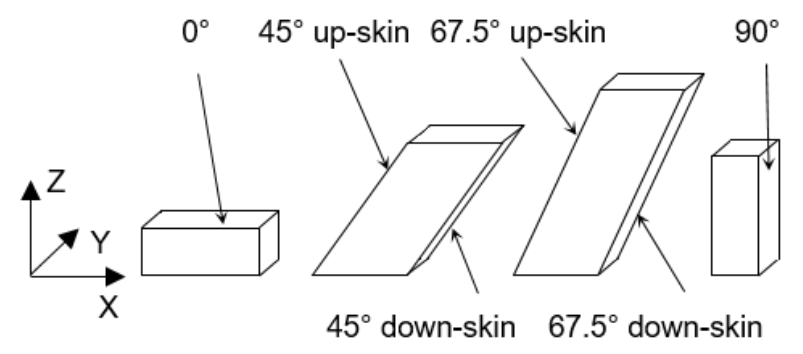

Fig. 1: Samples for the milling process.

The milling process was carried out on a 5-axis machining center (GROB G350T, GROB-Werke GmbH \& Co. KG., Mindelheim, Germany). A high-precision finishing mill was used for both roughing and finishing (SilverLine, High Accuracy Finish Milling Cutter, 50959060, CERATIZIT S.A., Mamer, Luxembourg). In Tab. 3, the cutting parameters of the milling process are listed.

The number of usage cycles of every cutting tool was set to a maximum of five finishing cycles, and high surface quality was ensured by connecting each phase of the milling process. First, the milling tool was used for finishing of one combination before it was used for the roughing process of the next one. 
Tab. 3: Cutting parameters for the milling process.

\begin{tabular}{ll}
\hline parameter & value \\
\hline tool diameter & $6 \mathrm{~mm}$ \\
number of teeth & 6 \\
spindle rotation speed (rpm) & $21221 / \mathrm{min}$ \\
feed rate per tooth (roughing) & $0.03 \mathrm{~mm} /$ tooth \\
feed rate per tooth (finishing) & $0.015 \mathrm{~mm} /$ tooth \\
radial depth of cut (roughing) & $0.3 \mathrm{~mm}$ \\
radial depth of cut (finishing) & $0.1 \mathrm{~mm}$ \\
\hline
\end{tabular}

The feed direction was oriented along the longitudinal axis of the component. The angle between the raw material surface and the final milled component amounted to $20^{\circ}$. During the roughing process, the surface was machined up to $0.1 \mathrm{~mm}$ of the target size. Subsequently, the remaining $0.1 \mathrm{~mm}$ were removed by finishing. The milling strategy is depicted in Fig. 2.

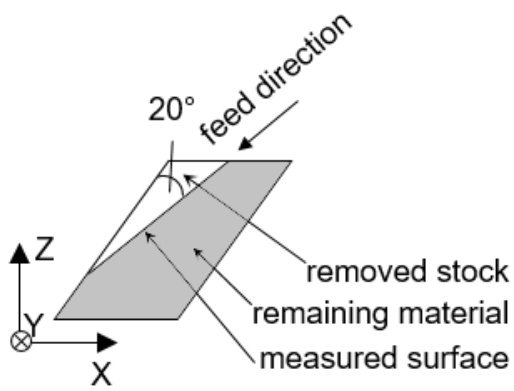

Fig. 2: Milling strategy, exemplarily depicted for combination number $\mathrm{M} 2$

\section{Measurement procedure}

Before and after machining, the surface quality was measured with a confocal laser scanning microscope VK-X1000 (Keyence Corporation, Osaka, Japan). An image of the milled surface was taken between the uncut surfaces with a $20 x$ magnification. Fig. 3 depicts this surface area. Next, each measured image was smoothed with the plane inclination filter. The measured distances were chosen according to DIN EN ISO 4288 [DIN EN ISO 4288] after using the plane inclination filter. Based on the measured lines, the waviness was observed. If the waviness exceeded a value of $10 \mu \mathrm{m}$, a waviness correction factor was applied.

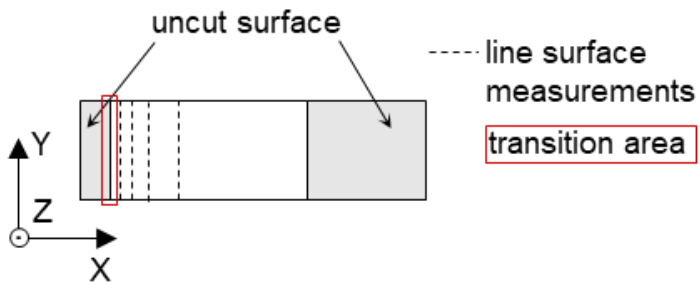

Fig. 3: Measurement strategy.

On the milled surface, four surface line measurements were arranged at a specific depth of material removal. These lines were aligned along the component cross axis. Four different material removals were investigated: $0.05 \mathrm{~mm}$, $0.10 \mathrm{~mm}, 0.20 \mathrm{~mm}, 0.50 \mathrm{~mm}$. The reference for the line measurements was the boundary, which indicates the transition from raw material to the milled surface. The boundary is located in the transition area. Due to the angle of material removal of $20^{\circ}$, these line measurements were placed at: $0.146 \mathrm{~mm}, 0.292 \mathrm{~mm}, 0.585 \mathrm{~mm}$, and $1.462 \mathrm{~mm}$, distance from the boundary, corresponding to the desired depths of material removal. The total length of every measured line was specified to $4.5 \mathrm{~mm}$. A pre-run section and a trailing section of $1 \mathrm{~mm}$ were excluded [DIN EN ISO 4288]. Due to the dimensions of the part, the evaluation length was equal to the sampling length. The measurement error is approximately $0.2 \mu \mathrm{m}$ [Keyence Deutschland]. On the samples kept in as-built condition, two measured sections along the component length axis were specified with a distance of $4.5 \mathrm{~mm}$. The total length of the measured line was $14 \mathrm{~mm}$, with the first and the last $3 \mathrm{~mm}$ as pre-run section and trailing section [DIN EN ISO 4288]. On all measurement lines, the arithmetical mean roughness $R_{a}$ was measured, and the mean and standard deviation of the four samples of each category were calculated.

\subsection{Mass finishing}

\section{Experimental procedure}

Based on the state of the art for mass finishing, two groups of experiments were defined in collaboration with the Doerfler \& Schmidt Praezisionsfinish GmbH (Kammerstein, Germany):

1. determination of the influence of the mass finishing process and processing time on the surface roughness and material removal, and

2. determination of the influence of process parameters: tank filling quantity and pellet size, on the surface roughness and material removal.

Tab. 4 details the factor combinations. Centrifugal disk finishing was carried out on an OTEC CF 50 with a rotational speed of $160 \mathrm{rpm}$. For vibratory finishing, a Roessler Trogvibrator Minor, with the maximum speed of $3000 \mathrm{rpm}$, was used. As the abrasive in the medium, a mix of RXF and RSG 10/20 ZS grinding pellets was used for $G 1$ to G9. For the experiments $\mathrm{G} 10$ to $\mathrm{G} 12$, respectively, RMB/D1 10/10 D, RMB/D1 15/15 D, and RMB/D1 20/20 D with a constant filling of $25 \mathrm{~kg}$ each were used. The duration was set to two hours for experiments G7 to G12. Doerfler \& Schmidt Praezisionsfinish GmbH (Kammerstein, Germany) carried out all mass finishing experiments. All abrasives were purchased from Rösler Oberflaechentechnik $\mathrm{GmbH}$ (Untermerzbach).

Tab. 4: Factor combinations for the mass finishing experiments.

\begin{tabular}{lll}
\hline $\begin{array}{c}\text { combination } \\
\text { number }\end{array}$ & \multicolumn{1}{c}{ machine } & second parameter \\
\hline G1 & centrifugal disk & duration: $1 \mathrm{~h}$ \\
G2 & centrifugal disk & duration: $2 \mathrm{~h}$ \\
G3 & centrifugal disk & duration: $3 \mathrm{~h}$ \\
G4 & vibratory & duration: $1 \mathrm{~h}$ \\
G5 & vibratory & duration: $2 \mathrm{~h}$ \\
G6 & vibratory & duration: $3 \mathrm{~h}$ \\
\hline G7 & centrifugal disk & filling: $7 \mathrm{~kg}$ \\
G8 & centrifugal disk & filling: $14 \mathrm{~kg}$ \\
G9 & centrifugal disk & filling: $28 \mathrm{~kg}$ \\
\hline G10 & centrifugal disk & pellets' size: $10 \mathrm{~mm}$ \\
G11 & centrifugal disk & pellets' size: $15 \mathrm{~mm}$ \\
G12 & centrifugal disk & pellets' size: $20 \mathrm{~mm}$ \\
\hline
\end{tabular}


Three samples for each set of parameters were manufactured to ensure the repeatability of the measurements. Since the point of interest was the comparison with the milling process, only external surfaces were studied. Therefore, the samples were designed as rectangular cuboids, resulting in the measured objects being flat surfaces. The chosen dimensions of $20 \times 20 \times 40 \mathrm{~mm}$ ensured that all samples were additively manufactured in one build job, keeping the additive manufacturing conditions similar for all the samples.

The samples were produced by FIT AG (Lupburg, Germany). All samples were built with the same orientation towards the protective gas, diagonal to the square profile (as seen in Fig. 4). After the additive manufacturing process, the samples were sawed off the build platform. The sawed surface designation is $\mathrm{E}$.

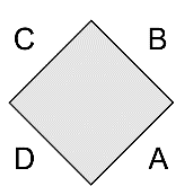

$$
\text { Protective gas }
$$

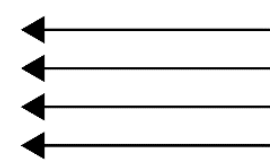

Fig. 4: Direction of the protective gas stream.

\section{Measurement procedure}

Two types of measurements, mass and surface roughness, were carried out to determine the influence of the mass finishing process parameters. The measurements were performed once before and once after the mass finishing process.

The mass of the samples was determined using a Gram FD410 scale (Gram Group, Barcelona, Spain). For statistical purposes, the measurements were repeated three times. The measurement error of the scale is $0.001 \mathrm{~g}$.

The length of the surface roughness measuring lines was defined according to DIN EN ISO 4288 [DIN EN ISO 4288] Based on the expected values for as-built components and finished components [Prüller 2018; EOS $\mathrm{GmbH}$ ], the filter $\lambda_{c}$ was set to $2.5 \mathrm{~mm}$ before and $0.8 \mathrm{~mm}$ after the mass finishing process. For each face, three lines parallel and three lines perpendicular to the build direction were measured. Initial measurements showed a similar roughness on surfaces with the same orientation towards the protective gas. Therefore, measurements were only carried out on surfaces with different orientations $(A, C$, and E). Before the mass finishing, a 3D optical profiler VR-3100 (Keyence Corporation, Osaka, Japan) was used to determine the roughness of each face. Its precision is $\pm 3 \mu \mathrm{m}$, which was sufficient for the expected as-built values. After the mass finishing, a tactile measuring instrument, Waveline W10 (JENOPTIK AG, Jena, Germany), was used to measure the roughness. Some measurements were taken with a laser scanning microscope, VK-X1000 (Keyence Corporation, Osaka, Japan), to verify the results of the Waveline W10. First, the measurement results of all devices were compared for a few samples. The laser scanning microscope and the Waveline W10 showed similar results, whereas the 3D optical profiler showed an offset of $2.5 \mu \mathrm{m}$ for $R_{a}$ and $20 \mu \mathrm{m}$ for $R_{z}$ to the results of the laser scanning-microscope. Since the laser scanning microscope has the highest precision, these offsets were added to the results of the 3D optical profiler before the analysis.

\section{RESULTS AND DISCUSSION}

\subsection{Milling}

\section{As-built surfaces}

The as-built samples had a surface roughness of $R_{a}$ between 23.9 and $57.0 \mu \mathrm{m}$. The as-built surfaces also showed partially melted particles, visible on the uncut area in Fig. 5.

\section{Influence of depth of material removal}

First, the influence of the depth of material removal on the surface roughness was analyzed. Fig. 6 depicts the mean of the arithmetic roughness $R_{a}$ with an increasing depth of material removal. For an increasing material removal, the mean roughness value and its deviations decrease, though the magnitude of decrease becomes smaller with an increasing depth of material removal. This holds true for both mean values and standard deviations. While the difference of surface roughness between the material removal of $0.05 \mathrm{~mm}$ and $0.10 \mathrm{~mm}$ for combination $\mathrm{M} 1$ amounts to $4.2 \mu \mathrm{m}$, the difference for a material removal of $0.10 \mathrm{~mm}$ and $0.20 \mathrm{~mm}$ decreases to $0.6 \mu \mathrm{m}$. Afterwards, the mean surface roughness remains stable, as the values are in the same range.

The surface roughness is caused by attached and not wholly melted particles, as shown in Fig. 5. If a material depth of $0.05 \mathrm{~mm}$ is removed, a significant reduction of the surface roughness is apparent. However, it is not sufficient to ensure stable surface quality, as the standard deviation is relatively high, with $R_{a}$-values between 12 and $73 \%$. Consequently, the necessary depth of material removal is $0.2 \mathrm{~mm}$

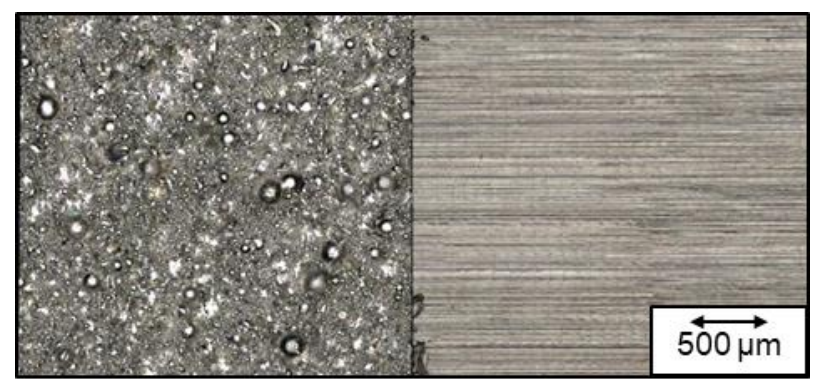

Fig. 5: Depiction of a milled M5 surface from uncut to finished surface.

\section{Influence of the orientation on the build platform}

Next, the influence of the orientation on the build platform on the surface roughness was determined. Overall, the asbuilt components with a $135^{\circ}$ orientation - combinations M7 to M12 - exhibit a slightly better surface quality than samples with a $45^{\circ}$ orientation (M1 to M6). At a material removal of $0.05 \mathrm{~mm}$, the effect is greatest. Here, the samples with $45^{\circ}$ orientation (M1 to M6) exhibit a higher average value than the ones with $135^{\circ}$ orientation angle (M7 to M12). The difference between the standard deviation proves the same. However, the size of the standard deviation is of the same order of magnitude, so that a clear difference is not detectable. Several causes might explain this behavior. Due to the orientation, the inert gas flow hits the surface differently, and the power of the laser might not suffice to melt the particles completely. This, in turn, might lead to a lowered surface quality. The scan strategy of the laser could also influence the surface roughness. For a higher material removal than $0.05 \mathrm{~mm}$, the difference in surface roughness values is no longer significant, as the values are in the same range. Therefore, the orientation angle does not influence the necessary depth of material removal. 


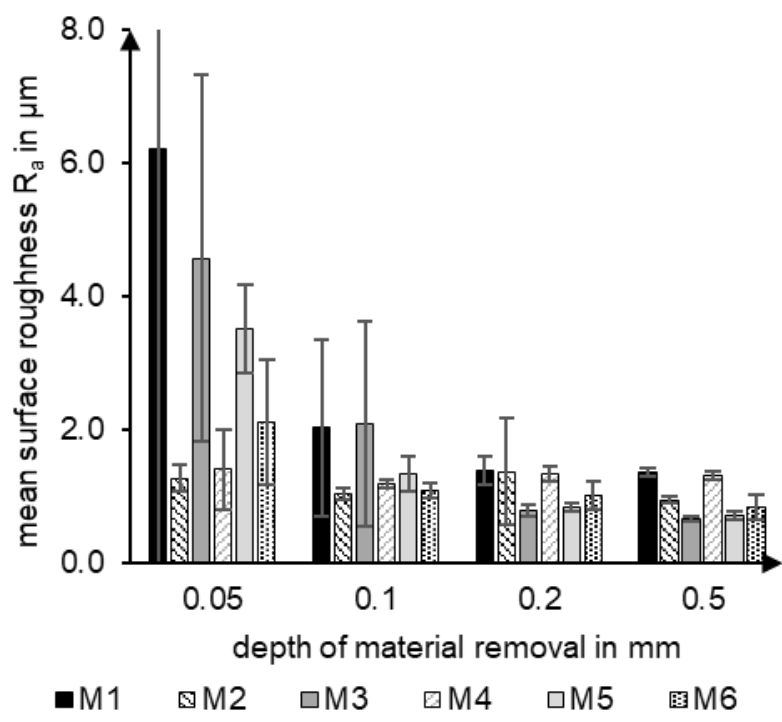

a) Factor combinations $M 1$ to $M 6$

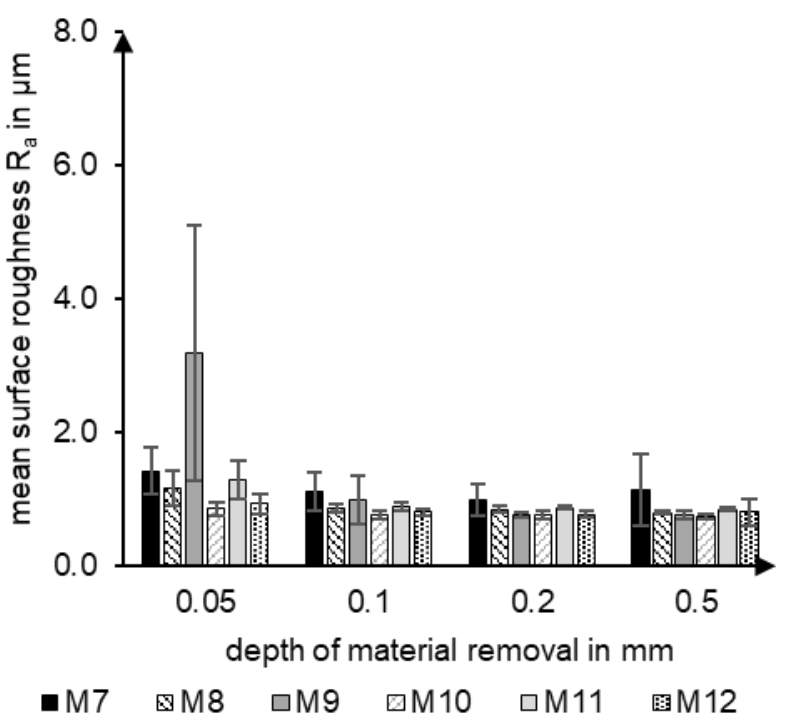

b) Factor combinations $M 7$ to $M 11$

Fig. 6: Surface roughness with an increasing depth of material removal, the values are depicted without the measurement error, the standard deviation for M1 is $\pm 6 \mu \mathrm{m}$.

\section{Influence of the surface angle orientation}

Then, the influence of the surface angle orientation - upskin or down-skin - was examined. Overall, the roughness values of the up-skin orientation (M4, M6, M10, M12) are lower than those of the down-skin orientation (M3, M5, M9, M11), with a depth of material removal of $0.05 \mathrm{~mm}$ and $0.10 \mathrm{~mm}$. From a material removal of $0.20 \mathrm{~mm}$ onward, the difference in roughness values is in the same range as the measurement errors of the microscope. Therefore, the surface quality is considered stable.

\section{Influence of surface angle and surface angle orientation}

Subsequently, the influence of the inclination angle and surface angle orientation on the surface roughness was investigated. On the as-built samples, the correlation between surface roughness and the inclination angle is unclear.

Overall, an inclination angle of $0^{\circ}(\mathrm{M} 1)$ results in the most unstable surface quality with a high standard deviation and relatively high surface roughness, even after milling. Samples show the most stable surface quality with an inclination angle of $90^{\circ}$ (M2 and M8). Here, the mean values, as well as the standard deviations, are at a lower level. If a choice between the two categories is possible, samples with an inclination of $90^{\circ}$ should be preferred. For $45^{\circ}$ and $67.5^{\circ}$ inclination angles (M3 to M6 and M9 to M12), the surface inclination is not the influencing factor, but instead, the surface angle orientation is. With the $45^{\circ}$ and $67.5^{\circ}$ up-skin orientation (M4, M6, M10, and M12), the same surface quality is achieved as with the $90^{\circ}$ orientation (M2 and M8), independent of the depth of material removal. On down-skin oriented surfaces (M3, M5, M9, and M11), the surface inclination angle of $45^{\circ}$ (M3 and M9) leads to lower quality than a $67.5^{\circ}$ inclination (M5 and M11). However, after the removal of more than $0.2 \mathrm{~mm}$, the influence of the inclination angle and the surface orientation becomes insignificant, since measured $R_{a}$-values are in the same range.
In summary, due to the inclination angle, a clear difference in the surface quality is detected for depths of material removal of $0.05 \mathrm{~mm}$ and $0.10 \mathrm{~mm}$. If the inclination angle is selectable and the depth of material removal should be reduced to a minimum, an inclination angle of $67.5^{\circ}$ and $90^{\circ}$ is preferable. At a higher material removal, a selection is unnecessary.

\subsection{Mass finishing}

\section{As-built surfaces}

The as-built samples showed a surface roughness of $R_{a}$ between 2.9 and $7.2 \mu \mathrm{m}$ and $R_{z}$ between 16.8 and $48.5 \mu \mathrm{m}$. The roughness was slightly higher in the build direction. These values are sufficient for force-fit and form-fit connections between surfaces, but some applications like sealing surfaces subjected to dynamic load require a lower roughness. With $R_{a}$ lower than $0.8 \mu \mathrm{m}$ and $R_{z}$ lower than $6.3 \mu \mathrm{m}$, the surface would be suitable for a large scope of applications [Labisch 2014]. Fig. 7 depicts the surface of a randomly chosen sample. Partially melted particles are visible. On the sawn surface substantial irregularities were detected.

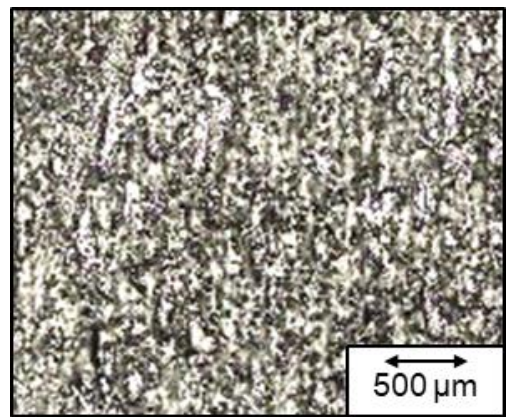

Figure 7: As-built surface on a random mass finishing sample. 


\section{Final surfaces and mass removal}

Fig. 8 depicts the measured reduction in sample mass after the mass finishing processes. Overall, parts processed by centrifugal disk finishing (G1 to G3 and G7 to G12) showed a higher mass removal than parts processed with vibratory finishing (G4 to G6). The removed material was 3 to 6 times higher for the centrifugal disk finishing than for the vibratory finishing. A longer duration of the mass finishing process (G1 to G3 and G4 to G6) led to a proportionally larger amount of material removal, while an increase in filling level (G7 to G9) resulted in a decrease in material removal. Larger pellets lead to higher material removal (G10 to G12).

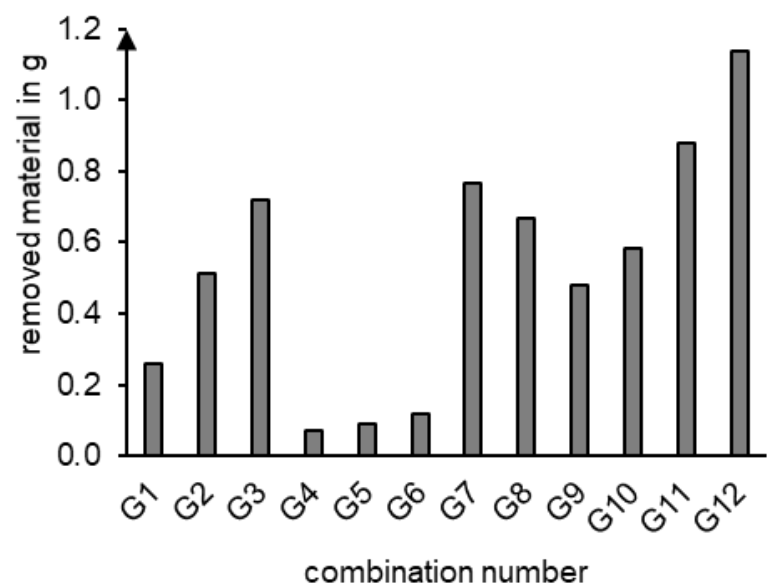

Fig. 8: Mass reduction after the mass finishing processes, values are displayed without the standard deviation between 1 and $13 \mathrm{mg}$.

Fig. 9 shows the surface quality of the components after the mass finishing process. After the centrifugal disk finishing process, the initial roughness was reduced by $75.5 \%$ after $1 \mathrm{~h}$ and up to $94.6 \%$ after $3 \mathrm{~h}$ (G1 to G3). In contrast, it was only reduced by 34.3 to $52.1 \%$ for the same operational times in the vibratory finishing machine (G4 to G6). Fig. 10 shows this contrast exemplarily on random samples of the G3 and G6 combinations. The lowest $R_{a}$ obtained after $3 \mathrm{~h}$ in the centrifugal disk machine is $0.37 \mu \mathrm{m}$. However, the roughness obtained after $2 \mathrm{~h}$ of mass finishing (G2), $R_{a}$ equal to $0.56 \mu \mathrm{m}$, is already low enough for a large scope of applications. The surface was visibly cleaned after $1 \mathrm{~h}$ with the centrifugal disk machine (G1). In the vibratory machine, most of the residual particles were removed only after $3 \mathrm{~h}$ (G6). The irregularities on the sawn surface were also removed only in the centrifugal disk process.

With each filling in the experiments G7 to G9, the roughness was reduced by around $90 \%$. The lowest roughness $R_{a}$ equal to $0.54 \mu \mathrm{m}$ and $R_{z}$ equal to $4.60 \mu \mathrm{m}$ was obtained with a filling of $14 \mathrm{~kg}$ (G8). However, the influence of the filling quantity on the surface roughness is not significant, and all of the final surface qualities are suitable for most applications (G7 to G9).

Like the roughness values, the aspect of the final surface does not vary depending on the filling. The surfaces were in all cases cleaned and smoothed. However, the substantial irregularities on the sawn surface were slightly better removed with the lowest filling quantity (G7). On the other hand, the material removal is inversely proportional to the filling quantity. It implies that the forces and the generated collision effect on the samples are higher when fewer grinding pellets are in the tank.

In the experiments with combinations $\mathrm{G} 10$ to $\mathrm{G} 12$, the roughness was reduced by 86.3 to $92.5 \%$. The lowest $R_{a}$ equal to $0.52 \mu \mathrm{m}$ and $R_{z}$ equal to $4.92 \mu \mathrm{m}$ were obtained with the smallest pellets (G10). $R_{a}$ equal to $0.99 \mu \mathrm{m}$ and $R_{z}$ equal to $7.66 \mu \mathrm{m}$ were obtained with the biggest pellets (G12). Consequently, the surfaces are suitable for more applications when they are processed with smaller pellets. In general, the sawn surfaces (E) are smoother than the surface $A$ and $C$. In contrast to surfaces $A$ and $C$, the irregularities on surface $E$ were removed better in experiment $\mathrm{G} 12$ than in the others. The material removal is also higher when bigger pellets are used. The results show that in this case, the mass removal is higher because of the higher grinding performance of big pellets, while the surface remains coarser.

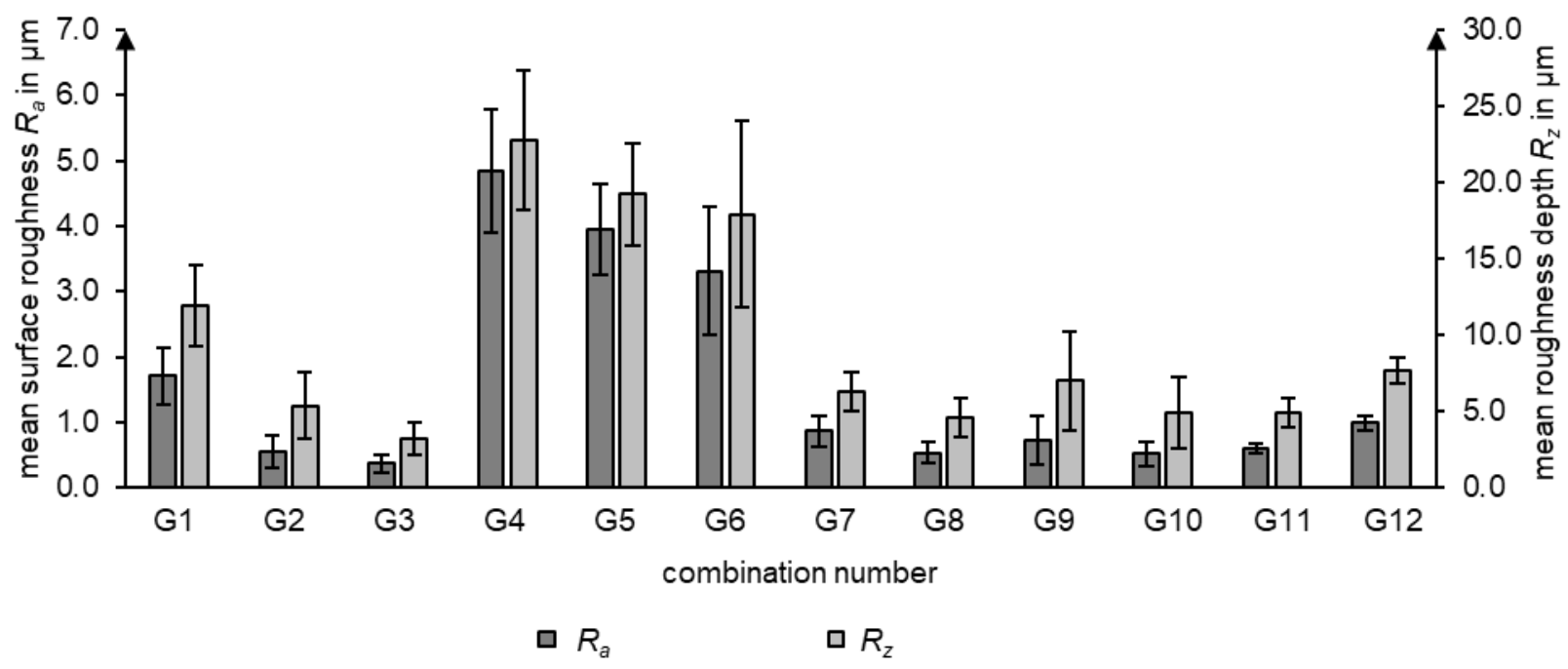

Fig. 9: Surface quality after the mass finishing processes 


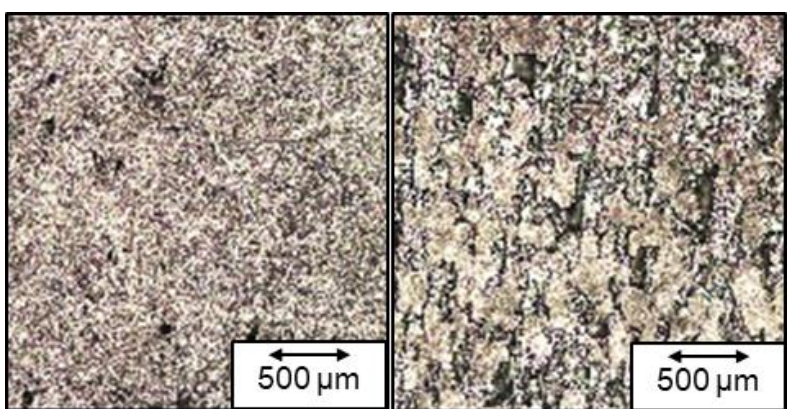

a) G3

b) G6

Figure 10: Surfaces after the mass finishing process.

\subsection{Assessment of the effectiveness for post- processing}

Overall, with the milling and the centrifugal disk finishing process, a surface quality sufficient for sealing surfaces was achieved. The surface quality after the vibratory finishing process was not sufficient for this kind of functional surface. For all three processes, the resulting surface quality was comparable to the state of the art.

With the milling process, a material removal of $0.2 \mathrm{~mm}$ was necessary to achieve a stable surface quality. For the mass finishing process, an approximated depth of materia removal $d_{a}$ is calculable, with the removed mass $M_{r}$, the density $\rho_{316 L}$, and the sample's surface $A$ :

$$
d_{a}=\frac{M_{r}}{\rho_{316 L} * A}
$$

For the factor combinations leading to a surface quality comparable to the milling process (G2, G3, and G7 to G11), the depth of material removal was approximately between $0.015 \mathrm{~mm}$ and $0.027 \mathrm{~mm}$, much lower than for the milling process. These depths of material removal are in the range of the beforehand measured $R_{z}$ values. Likely, the mass finishing processes remove the peaks of the surface topography, resulting in lower surface roughness.

The determined necessary depth of material removal of $0.2 \mathrm{~mm}$ for the milling process is much higher. The higher surface roughness of the as-built samples for the milling process is possibly the reason. As the samples for the milling and mass finishing process were manufactured in two batches, a change of the machine used for production, process parameters, or powder might be the cause. However, using a rough approximation of $R_{z}$ to $R_{a}$ of $4: 1$ [Schuetz 2021], it is reasonable to assume that the depth of material removal is in the range of the $R_{z}$ value as well. Conclusively, the necessary depth of material removal does not significantly depend on the specific post-processing process but on the surface quality of the as-built components.

Mass finishing processes aim to enhance the surface quality without focusing on form or dimension [Klocke 2017]. Therefore, if form tolerances are of no significance and the surface roughness is within the range for formfitting or force-fitting applications, centrifugal disk finishing is a post-processing option to achieve functional surfaces. The option of processing in batches enhances its economic value. In contrast, if form tolerances are of significance, or the surface quality of the as-built samples is low, milling should be used for post-processing.

\section{SUMMARY AND OUTLOOK}

Within this study, three traditional machining processes: milling, vibratory finishing, and centrifugal disk finishing, were assessed as post-processing operations for PBF-LB/M-manufactured components. For the assessment, the achievable surface quality and the necessary depth of material removal were considered. Key findings of the research include:

- Independent of the part orientation on the build platform, surface angle, and surface orientation, a stable surface quality is achievable with the milling process at a material removal of $0.2 \mathrm{~mm}$. Therefore, the necessary depth of material removal in the milling process is $0.2 \mathrm{~mm}$.

- $\quad$ The surface quality after the centrifugal disk finishing is higher than after the vibratory finishing.

- The processing time does influence the surface quality after centrifugal disk finishing significantly, while tank filling quantity and pellet size are not as significant.

- The necessary depth of material removal seems to correlate with the surface quality of the as-built components.

One limiting factor for the findings is the different surface quality of the as-built components. The surface roughness of the milling samples was initially much higher than the surface roughness of the mass finishing samples. A clear economic comparison of the three processes is therefore not possible. To design economic process chains, further experiments on this subject should be carried out. Furthermore, only the surface roughness was examined. Depending on the process parameters, the parts might have a porous layer close to the surface. It should be studied whether this layer was removed or compressed during the mass finishing to form a fully dense surface. At the same time, materials tend to anisotropic mechanical behavior [Ni 2017; Chen 2018] due to the PBF-LB/Mprocess, which has to be considered for process applications. Finally, it is unclear whether these results can be applied to other materials as well. Some material forms slags during the additive manufacturing process. The necessary depth of material removal might then not correlate with the surface quality of the as-built components, which should also be examined in the future.

\section{ACKNOWLEDGEMENTS}

This research was funded by the Bavarian Research Foundation (Bayerische Forschungsstiftung) in the research project ShapeAM (Capability of additive manufacturing technologies for the production of functional components with high quality requirements for industrial use) within the funding program "High Technologies for the $21^{\text {st }}$ Century", grant number AZ-1307-17. The authors would like to thank the Bavarian Research Foundation and the project partners for their support and cooperation.

The authors would also like to thank Ms. Christina Haeussinger for her ideas and help in the preparation and execution of the milling experiments.

\section{REFERENCES}

[Afkhami 2021] Afkhami, S. et al. Effects of manufacturing parameters and mechanical post-processing on stainless steel 316L processed by laser powder bed fusion. Materials Science and Engineering: A, 2021, Vol. 802, p. 140660. ISSN 09215093

[Calleja-Ochoa 2021] Calleja-Ochoa, A. et al. A New Approach in the Design of Microstructured Ultralight Components to Achieve Maximum Functional Performance. Materials (Basel, 
Switzerland), 2021, Vol. 14, No. 7. ISSN 19961944

[Chen 2018] Chen, Z. et al. Anisotropy of nickel-based superalloy K418 fabricated by selective laser melting. Progress in Natural Science: Materials International, 2018, Vol. 28, No. 4, pp. 496504. ISSN 10020071

[Domblesky 2003] Domblesky, J. et al. Investigation of vibratory bowl finishing. International Journal of Production Research, 2003, Vol. 41, No. 16, pp. 3943-3953. ISSN 0020-7543

[Elangeswaran 2019] Elangeswaran, C. et al. Effect of post-treatments on the fatigue behaviour of 316 L stainless steel manufactured by laser powder bed fusion. International Journal of Fatigue, 2019, Vol. 123, pp. 31-39. ISSN 01421123

[EOS GmbH] EOS GmbH EOS StainlessSteel 316L: Material Data Sheet. Available from $<$ https://www.eos.info/03_system-relatedassets/material-related-contents/metalmaterials-and-examples/metal-materialdatasheet/stainlesssteel/material_datasheet_eo s_stainlesssteel_316l_en_web.pdf>

[DIN EN ISO 4288] European Committee for Standardization. DIN EN ISO 4288, November 1997

[Gibson 2015] Gibson, I. et al. Additive Manufacturing Technologies, 2015

[Hitzler 2017] Hitzler, L. et al. Position dependent surface quality in selective laser melting. Materialwissenschaft und Werkstofftechnik, 2017, Vol. 48, No. 5, pp. 327-334. ISSN 09335137

[Kaynak 2018] Kaynak, Y. and Kitay, O. Porosity, Surface Quality, Microhardness and Microstructure of Selective Laser Melted 316L Stainless Steel Resulting from Finish Machining. Journal of Manufacturing and Materials Processing, 2018, Vol. 2, No. 2, p. 36

[Kaynak 2019] Kaynak, Y. and Kitay, O. The effect of postprocessing operations on surface characteristics of 316L stainless steel produced by selective laser melting. Additive Manufacturing, 2019, Vol. 26, pp. 84-93. ISSN 22148604

[Keyence Deutschland] Keyence Deutschland VK-X1000 Datasheet. [online] [16.08.2021]. Available from $<$ https://www.keyence.de/products/microscope/l aser-microscope/vk-x100_x200/models/vk$\mathrm{x} 1000 />$

[Kleszczynski 2015] Kleszczynski, S. et al. Position dependency of surface roughness in parts from laser beam melting systems. In: D.L. Bourell, ed. Proceedings // 26th Annual International Solid Freeform Fabrication Symposium - an
Additive Manufacturing Conference : August 10 - 12, 2015, \[Austin, Texas $\mid] /$ The Universtiy of Texas at Austin, Austin, Texas, USA ; ed. by David L. Bourell ... ; Pt. 1, Annual International Solid Freeform Fabrication Symposium - an Additive Manufacturing Conference; 26. Austin, Tex.: University of Texas at Austin. 2015

[Klocke 2014] Klocke, F. et al. Cutting with Geometrically Defined Cutting Edges. In: T. Mang, ed. Encyclopedia of Lubricants and Lubrication. Berlin, Heidelberg: Springer Berlin Heidelberg. 2014, pp. 338-361. ISBN 978-3-642-22646-5

[Klocke 2017] Klocke, F. Fertigungsverfahren 2 [Manufacturing processes 2], 2017

[Kumbhar 2018] Kumbhar, N.N. and Mulay, A.V. Post Processing Methods used to Improve Surface Finish of Products which are Manufactured by Additive Manufacturing Technologies: A Review. Journal of The Institution of Engineers (India): Series C, 2018, Vol. 99, No. 4, pp. 481487. ISSN 2250-0545

[Labisch 2014] Labisch, S. and Weber, C. Technisches Zeichnen [Technical drawing], 2014

[Loeber 2013] Loeber, L. et al. Comparison of different post processing technologies for SLM generated 316l steel parts. Rapid Prototyping Journal, 2013, Vol. 19, No. 3, pp. 173-179. ISSN 1355-2546

[Ni 2017] Ni, M. et al. Anisotropic tensile behavior of in situ precipitation strengthened Inconel 718 fabricated by additive manufacturing. Materials Science and Engineering: A, 2017, Vol. 701, pp. 344-351. ISSN 09215093

[Prueller 2018] Prueller, H. Praxiswissen Gleitschleifen [Practical knowledge of mass finishing processes], 2018

[Schuetz 2021] Schuetz, G. Surface Texture From Ra To Rz. [online] [21.04.2021]. Available from $<$ https://www.mahr.de/enus/Services/Production-metrology/Knowhow/Gaging-Tips/Surface-MeasurementGaging-Tips/?ContentID=19020\&Overview=0>

[Sun 2019] Sun, Y. et al. Surface finish and properties enhancement of selective laser melted $316 \mathrm{~L}$ stainless steel by surface mechanical attrition treatment. Surface and Coatings Technology, 2019, Vol. 378, p. 124993. ISSN 02578972

[Thomas 2009] Thomas, D. The Development of Design Rules for Selective Laser Melting. Ph.D. Thesis. University of Wales Institute, 2009

[Zhang 2019] Zhang, J. et al. Surface quality and material removal in magnetic abrasive finishing of selective laser melted 316L stainless steel. Journal of Manufacturing Processes, 2019, Vol. 45, pp. 710-719. ISSN 15266125 